\title{
Inductive analysis of the deflection of a beam truss allowing kinematic variation
}

\author{
Mikhail Kirsanov ${ }^{1, *}$,Evgeny Komerzan ${ }^{1}$ and Olesya Sviridenko ${ }^{1}$ \\ ${ }^{1}$ National Research University "Moscow Power Engineering Institute", Krasnokazarmennaya 14, \\ Moscow, 111250, Russia
}

\begin{abstract}
A scheme of a statically determinate planar truss is proposed and an analytical calculation of its deflection and displacement of the mobile support are obtained. The forces in the rods from the external load, uniformly distributed over the nodes of the lower or upper belt, are determined by the method of cutting out nodes using the computer mathematic system Maple. In the generalization of a number of solutions of trusses with a different number of panels to the general case, the general terms of the sequence of coefficients in the formulas are found from solutions of linear homogeneous recurrence equations. To compose and solve these equations, Maple operators were used. In the process of calculation it was revealed that for even numbers of panels in half the span, the determinant of the system of equations degenerates. This corresponds to the kinematic degeneracy of the structure. The corresponding scheme of possible speeds of the truss is given. The displacement was determined by the Maxwell-Mohr's formula. The graphs of the obtained dependences have appreciable jumps, which in principle can be used in the selection of optimal design sizes.
\end{abstract}

\section{Introduction}

Numerical methods for calculating rod systems [1-5] have an alternative. With the advent of mathematical systems (Maple, Mathematica, Derive, Reduce) producing symbolic operations, it became possible to derive finite formulas for various characteristics of the stress-strain state of the trusses. However, formulas for concrete designs with a certain number of rods and a certain configuration are not very interesting for practical engineers. For example, when choosing a scheme for a projected design, it is desirable to have formulas that are derived for an arbitrary number of panels. Then the engineer by the formulas can pick up not only the sizes of a design, but also the optimum number of panels. Such problems arise when solving optimization problems [6-9]. In this paper, we propose an inductive approach for deriving the formulas for the dependence of the planar truss bending on the number of panels. Previously, this approach was used in solving problems of planar [10-13] and spatial [14-16] trusses.

\footnotetext{
*Corresponding author: c216@ya.ru
} 


\section{Truss and Methods}

Consider a beam truss with a double lattice (Fig. 1)/ The height of truss is $2 h$ and a span length of $L=2 a n$ / The are $m=8 n+10$ rods, including three support rods. A feature of the truss grille is the central truncated stance.

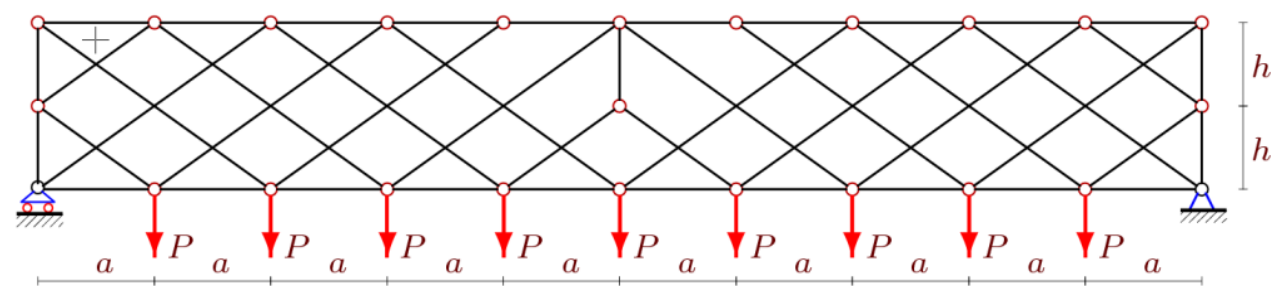

Fig. 1. Truss, load on the lower belt $n=5$

Calculation of forces is made by cutting out the nodes in the system of symbolic mathematics Maple. For this purpose, a matrix of directing cosines of all forces in the rods is compiled. The solution of the system of equations gives the values of the forces necessary to calculate the deflection according to the Maxwell-Mohr's formula. It was noted that for an even number of panels in half the span, the determinant of the system of equilibrium equations degenerates. This indicates an instantaneous variability of the design, which is completely unacceptable in practice. As a confirmation of this fact, we present a picture of the distribution of the possible velocities of the nodes of the truss (Fig. 2). It was noted that for an even number of panels in half the span, the determinant of the system of equilibrium equations degenerates. This indicates an instantaneous variability of the design, which is completely unacceptable in practice. As a confirmation of this fact, we present a picture of the distribution of the possible velocities of the nodes of the truss (Fig. 2). Obviously, $u / a=v / h$. This follows from the consideration of the centers of instantaneous velocities, which in particular are in the supports of the truss.

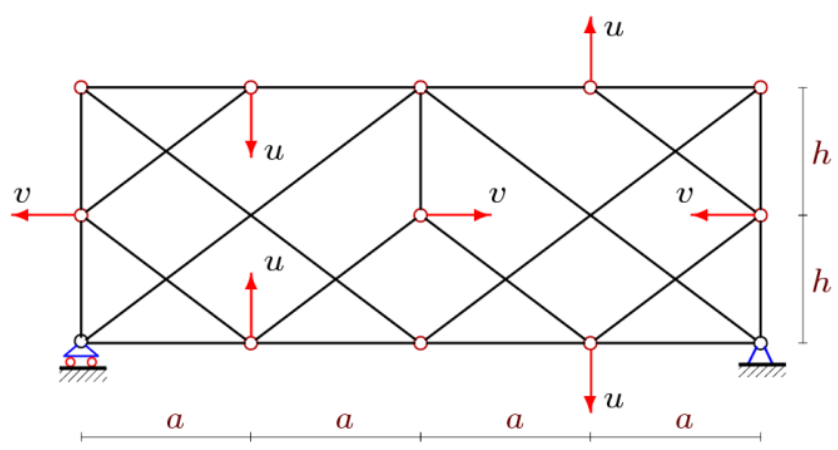

Fig. 2. Distribution of the possible velocities, $n=2$

Proceeding from this, we will consider only truss with an odd number of panels in half the span. We introduce a new parameter $n=2 k-1$. The displacement of the middle node of the lower belt is determined by the Maxwell-Mohr's formula

$$
\Delta=\sum_{i=1}^{m-3} S_{i}^{(P)} S_{i}^{(1)} l_{i} /(E F) .
$$


It is indicated: $l_{i}$ - the length of the rods, $S_{i}^{(P)}$ - the forces in the rods from the given load, $S_{i}^{(1)}$ - the forces from the unit force applied to the knot of the lower belt in the middle of the span, $E$ is the modulus of elasticity of the rods, and $F$ is the area of their cross sections.

\section{Results}

The solution for trusses with an arbitrary number of panels has the form

$$
\Delta=P\left(C_{1} a^{3}+C_{2} c^{3}+C_{3} h^{3}\right) /\left(2 h^{2} E F\right) .
$$

where $c=\sqrt{a^{2}+h^{2}}$. The calculation of a series of trusses reveals sequences of coefficients for $a^{3}, h^{3}, c^{3}$. Operators of the Maple system rgf_findrecur and rsolve from the solution of recurrence equations give the following patterns

$$
\begin{aligned}
& C_{1}=\left(20 k^{4}-40 k^{3}+16 k^{2}-2\left(6(-1)^{k}+1\right) k+3(-1)^{k}+3\right) / 6, \\
& C_{2}=\left(2 k^{2}-4\left(2(-1)^{k}+1\right) k+3(-1)^{k}+3\right) / 2, \\
& C_{3}=2\left(\left(2-(-1)^{k} k-(-1)^{k}-1\right.\right.
\end{aligned}
$$

For the coefficient $C_{1}$, a recurrence equation of the seventh order $C_{1, k}=3 C_{1, k-1}-C_{1, k-2}-5 C_{1, k-3}+5 C_{1, k-4}+C_{1, k-5}-3 C_{1, k-6}+C_{1, k-7}$.

This equation was solved with initial conditions

$$
C_{1,1}=1, C_{1,2}=7, C_{1,3}=119, C_{1,4}=461, C_{1,5}=1325 \text {. }
$$

The coefficient $C_{2}$ is obtained from the solution the linear equation $C_{2, k}=C_{2, k-1}+2 C_{2, k-2}-2 C_{2, k-3}-C_{2, k-4}+C_{2, k-5}$.

Expression (1) with coefficients (2) gives the solution of the problem posed for the load along the lower belt of the truss.

Similarly, for the load on the upper belt of the truss (Figure 3), we obtain the deflection in the form (1) and the similar coefficients

$$
\begin{aligned}
& C_{1}=\left(20 k^{4}-40 k^{3}+16 k^{2}+2\left(6(-1)^{k}+5\right) k+3(-1)^{k+1}-3\right) / 6, \\
& C_{2}=\left(2 k^{2}+8(-1)^{k} k-3(-1)^{k}+1\right) / 2, \\
& C_{3}=2\left(2-(-1)^{k}\right) k+3(-1)^{k}-1 .
\end{aligned}
$$

Coefficients are obtained from the same recurrence equations, but with other initial data $C_{1,1}=-1, C_{1,2}=17, C_{1,3}=113, C_{1,4}=483, C_{1,5}=1315$.

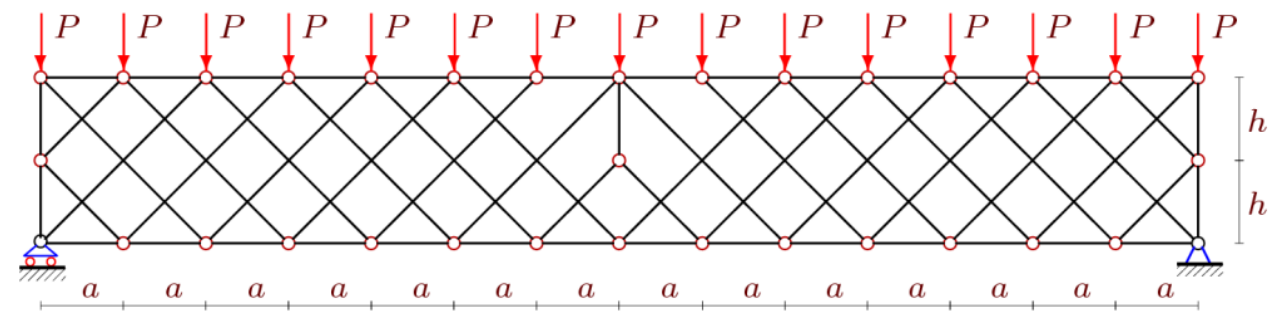

Fig. 3. Truss, load on the upper belt $n=7$ 
The simplest recurrence equations and their solutions will be for the case of loading by force in the middle of the span (Figure 4):

$$
\begin{aligned}
& C_{1}=(2 k-1)\left(2 k^{2}-2 k+3\right) / 3, \\
& C_{2}=3(2 k-1), \\
& C_{3}=5-(-1)^{k} .
\end{aligned}
$$

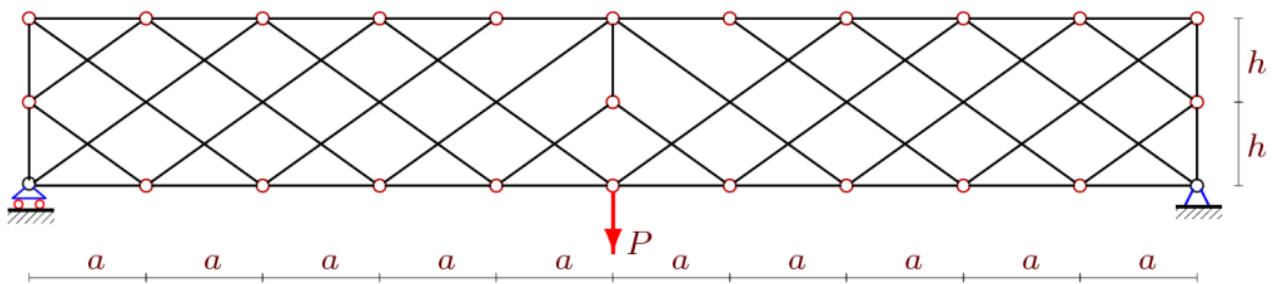

Fig. 4. Truss, load on the center, $n=5$

\section{Discussion}

Consider for example the graph of the dependence of the relative (dimensionless) deflection $\Delta^{\prime}=\Delta E F /\left(P_{0} L\right)$ on the number of panels for the case of loading on the upper belt. Figure 5 shows the curves of the obtained dependence at $L=2 n a=100 m, P_{0}=P(2 n+1)$. The curves behave almost chaotically. At the beginning of the graph, they fall sharply, then increase in an abrupt way. The slope of the asymptote is obvious from the formula (1)

$$
\lim _{k \rightarrow \infty} \Delta^{\prime} / k=h /(8 L) \text {. }
$$

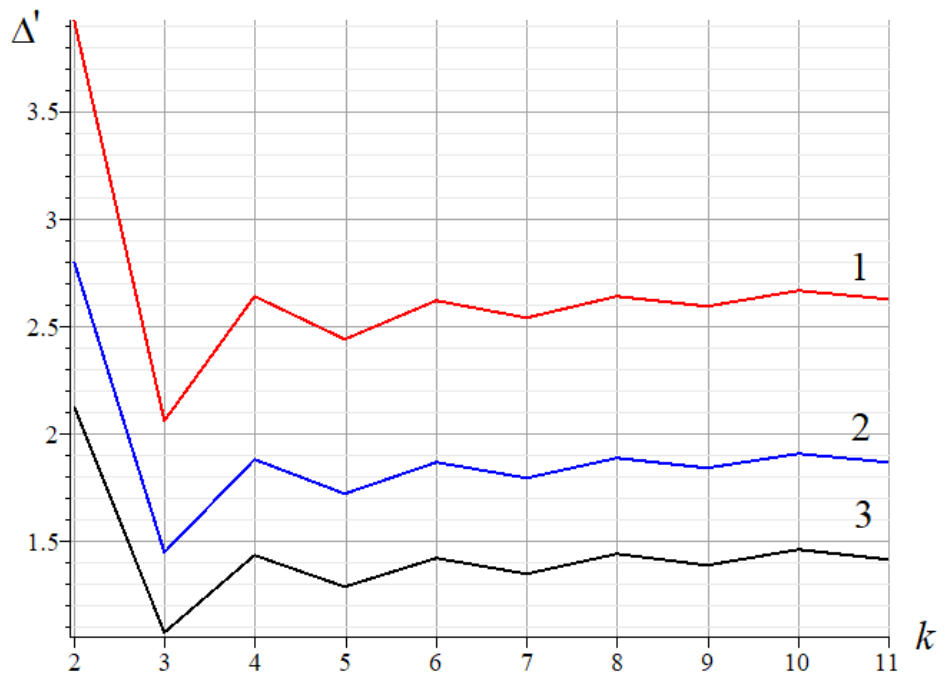

Fig 5. Dependence of the dimensionless deflection on the number of panels during loading on the upper belt. $1-h=5.00 \mathrm{~m}, 2-h=6.00 \mathrm{~m}, 3-h=7.00 \mathrm{~m}$ 
Figure 6 clearly shows the forces in the rods, referred to the magnitude of the force ( $L=2 n a=150 \mathrm{~m}, h=10 \mathrm{~m}$ ). The thicknesses of the lines are proportional to the magnitude of th $S_{i}^{(P)} / P, i=1, . ., m$, the blue color is divided into compressed rods, the red ones are stretched. It can be seen that the main load is carried by the belts, the forces in the bars of the lattice are significantly (by an order of magnitude) smaller. At the same time as expected, the descending rods were stretched.

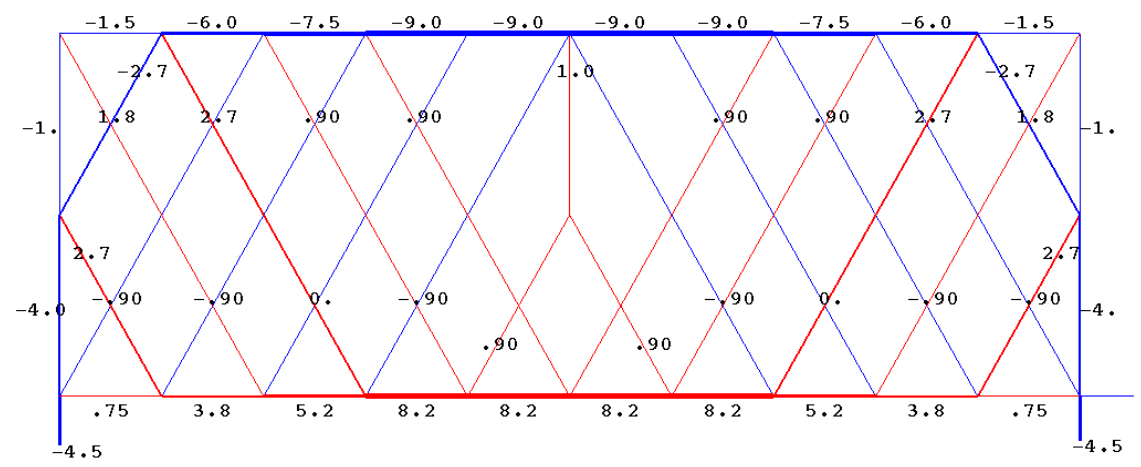

Fig 6. The distribution forces in the rods of the truss when the lower belt is loaded, $n=5$

When the number of panels is changed, the picture of the distribution of forces remains almost unchanged (Figure 7), with the exception of the "trefoil" rods in the middle of the span, where the signs of effort change.

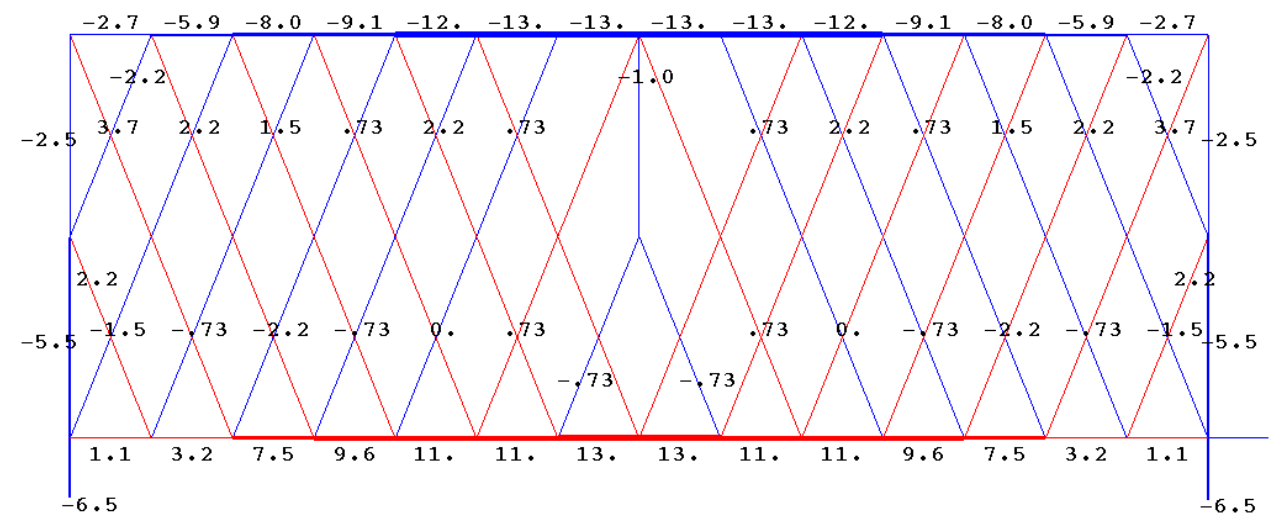

Fig 7. The distribution forces in the rods of the truss when the lower belt is loaded, $n=7$

In addition to the size of the vertical deflection, the deformability of the truss can be estimated from the horizontal displacement of the mobile support from the action of the loads. The horizontal displacement of the support is determined by the Maxwell-Mora formula

$$
\delta_{A}=P \sum_{i=1}^{m-3} S_{i}^{(P)} S_{i}^{(2)} l_{i} /(E F)
$$

where $S_{i}^{(2)}$ — the forces from the unit horizontal force applied to the left support. Omitting the intermediate calculations, we give the formula for the displacement of the support from the action of loads along the upper belt (Figure 1) 


$$
\delta_{A}=3 P a^{2}\left(16 k^{3}-24 k^{2}+3(-1)^{k}+20 k-3\right) /(6 h E F) .
$$

When the lower belt is loaded, the displacement is similar

$$
\delta_{A}=3 P a^{2}\left(16 k^{3}-24 k^{2}-3(-1)^{k}-4 k+3\right) /(6 h E F) .
$$

\section{Conclusions}

A feature of the proposed construction of the truss is a shortened middle stand. The fastening of this rack in the form of a "trefoil" made it possible to obtain a statically determinate scheme of the truss, for which analytical methods of calculation are available. Three solutions of the deflection problem for three types of load and the solution of the problem of shear support are obtained. Kinematic analysis revealed a latent design defect, manifested in the degeneracy of the determinant of equilibrium equations for even numbers of panels. The solutions found are of a rather simple form and can be used to test numerical results. This is especially important for trusses with a large number of panels, where numerical methods begin to malfunction due to the obvious accumulation of rounding errors. A brief review of some papers using the described induction method for the analytical calculation of trusses is given in [19].

\section{References}

1. A.B. Bondarev, A. Yugov, Magazine of Civil Engineering, 1, 60 (2016)

2. M. Y. Baranovskiy, V. A. Tarasov, Construction of Unique Buildings and Structures 7, 92 (2014)

3. A.V. Alekseytsev, N.S. Kurchenko, Magazine of Civil Engineering, 5, 3 (2017)

4. I.N. Serpik, A.V. Alekseytsev, P.Yu. Balabin, N.S. Kurchenk, Magazine of Civil Engineering, 8, 181 (2017)

5. T. Saknite, D. Serdjuks, V. Goremikins, L. Pakrastins, N.I. Vatin, Magazine of Civil Engineering, 4, 26 (2016)

6. A. Kaveh, V. R. Mahdavi, Structural Engineering and Mechanics 5, 847 (2015)

7. A. Kaveh, A. Zolghadr, Applied Soft Computing Journal, 5, 2727 (2013)

8. A. Ahrari, D. Kalyanmoy, Computers \& Structures 164, 127 (2016)

9. I.N. Serpik, A.V. Alekseytsev, Magazine of Civil Engineering, 1, 14 (2016)

10. M.N. Kirsanov, N. Zaborskaya, Magazine of Civil Engineering, 3, 61 (2017)

11. M.N. Kirsanov, Magazine of Civil Engineering,1, 33 (2016)

12. M.N. Kirsanov, Magazine of Civil Engineering, 5, 58 (2015)

13. M.N. Kirsanov, Magazine of Civil Engineering, 4, 38 (2015)

14. M.N. Kirsanov, Magazine of Civil Engineering, 4, 52 (2016)

15. M.N. Kirsanov, Magazine of Civil Engineering, 8, 58 (2017)

16. M.N. Kirsanov, Magazine of Civil Engineering, 1, 32 (2015)

17. D.V. Tinkov, Magazine of Civil Engineering, 1, 25 (2016)

18. M.N. Kirsanov, Magazine of Civil Engineering, 3, 3 (2015)

19. D.V. Tinkov, Magazine of Civil Engineering, 5, 66 (2015) 